\author{
Annals of Cancer Research and Therapy \\ Volume 10, pages 89-92 \\ Copyright $\odot 2002$ by \\ PJD Publications Limited \\ Research Communications \\ Westbury, NY 11590-0966 USA
}

\title{
DESIGN AND METHODOLOGY OF META-ANALYSIS TO SHOW THE EFFICACY OF OK-432
}

\author{
Junichi Sakamoto 1), Satoshi Teramukai 2), and Yasuo Ohashi 3)
}

1) Department of Epidemiological \& Clinical Research Information Management, Kyoto University

2) Department of Clinical Trial Management, Translational Research Center, Kyoto University

3) Department of Biostatistics / Epidemiology and Preventive Health Science, University of Tokyo, Japan

For The Japanese Meta-Analysis Group in Cancer in the Japanese Society of Strategies for Cancer Research and Therapy.

Key Words: Adjuvant therapy, cancer, drug efficacy, OK-432, imunochemotherapy, picibanil.

\section{Introduction}

The Japanese Meta-Analysis Group in Cancer was formed in the Japanese Society of Strategies for Cancer Research and Therapy to evaluate the effect of adjuvant therapy of cancer using meta-analysis. Performance of a meta-analysis to review all relevant trials is considered to be one of the best methods with a reasonable chance of detecting small, but worthwhile, clinical benefits.

We previously reported the therapeutic efficacy of oral 5-FU and related agents against curatively resected colorectal cancer (Sakamoto, Hamada et al., 1999).

Address correspondence and reprints requests to: Junichi Sakamoto, M.D., Department of Epidemiological \& Clinical Research Information Management, Kyoto University, Graduate School of Medicine, Yoshida-Konoecho, Sakyo-ku, Kyoto 606-8315, Japan. TEL: 81-75-752-1511; FAX: 81-75-752-1518; e-mail: sakamoto@pbh.med.kyoto-u.ac.jp 


\section{Object}

OK-432 (Picibanil) is a lyophilized preparation of a low-virulence group A Streptococcus pyogenes ( $\mathrm{Su}$ strain) of human origin that has been inactivated by treatment with benzyl-penicillin and heat. In 1975, The Japanese Ministry of Health and Welfare approved the use of OK-432 as an anticancer agent (Kurokawa, Hattori et al., 1972).

The therapeutic efficacy of imunochemotherapy with $\mathrm{OK}-432$ is reassessed in patients with curatively resected gastric cancer using a meta-analysis based on individual patient data from randomized clinical trials. In this analysis, the administration of OK-432 is systemic, i.e. intra-muscular or intra-dermal.

\section{Materials and Methods}

\section{1) Literature search}

A search will be performed of the MEDLINE data base and presentations at meeting, and inquiries will be made to industry, for the following items: OK-432 (Picibanil), randomized trial, gastric cancer, published since 1980 with an English or Japanese abstract. The reports found are summarized independently by two of the investigators, using a standardized summary form. The information is provided about the number of trials identified, included, and excluded and the reasons for excluding them (Moher, Cook et al., 1999).

2) Funnel plot

Publication bias is assessed using the funnel plot approach recommended for meta-analysis with few studies. The funnel plot is drawn up with sample size and odds ratios of individual trials (Felson, 1992).

\section{Statistical analysis}

\section{a) Primary analysis}

The endpoint of interest is the overall 5-year survival on an intent-to-treat basis. To assess the quality of trials, the analysis was carried out for trials using central randomization or envelope-method randomization. Individual patient data were collected from inquiries for trialist of each study. Analyses were performed using SAS procedure, including LIFETEST and PHREG. The relative risk ratios and their 95\% confidence 
intervals were calculated by Cox's proportional hazard model, with treatment effect being stratified by study

b) Prospective analysis in selected subgroups

Subgroup analysis of individual prognostic factors will be performed. The beneficial effect of immunochemotherapy with OK-432 was particularly relevant in patients with advanced gastric cancer patients. The trials that reported the efficacy were either oral administration (Kyoto research group for digestive organ surgery, 1992) or intratumoral administration (Tanaka, Gouchi et al., 1994) of OK-432 in patients with positive lymph node metastasis. The effect of intra peritoneal administration of OK-432 was reported in malignant ascites (Torisu, Katano et al., 1983), and peritoneal infiltration of immunological effecter cells after the preoperative systemic administration of OK-432 was demonstrated (Omote, Fujimoto et al., 1990). Therefore, a subgroup of patients who had advanced tumors with histological tumor invasion to serosal wall could be a good candidate to assess the beneficial effects of OK-432 and will be analyzed in a meta-analysis of systemic OK-432 treatment. The effect of splenectomy (Kyoto research group for digestive organ surgery, 1992) or delayed-type hypersensitivity, e.g. skin test with bacterial antigen (Watanabe, Iwa et al., 1984), must also be examined to improve the evaluation of immunochemotherapy.

\section{Conclusion}

Our group has started to analyze the treatment efficacy of adjuvant immunochemotherapy with $\mathrm{OK}-432$ for resected gastric cancer patients. The results of those meta-analysis will be published after the completion of all the planned study.

\section{References}

Felson DT. (1992). Bias in meta-analytic research. J Clin Epidemiol, 45:885-892.

Kurokawa T, Hattori T, Furue H. (1972). Clinical experiences with the streptococcal anticancer preparation, OK-432 (NSC-B116209). Cancer Chemother Rep, 56:211-220. 
Kyoto research group for digestive organ surgery. (1992). A comprehensive multi-institutional study on postoperative adjuvant immunotherapy with oral streptococcal preparation OK-432 for patients after gastric cancer surgery. Ann Surg, 216:44-54.

Moher D, Cook DJ, Eastwood S, Olkin I, Rennie D, Stroup DF. (1999). Improving the quality of reports of meta-analysis of randomized controlled trials: the QUOROM statement. Lancet, 354:1896-1900.

Omote K, Fujimoto T, Mai M, Sakai S. (1990). Analysis of immunological parameters of gastric cancer patients after preoperative administration of BRM. Biotherapy, 4:868-873.

Sakamoto J, Hamada C, Kodaira S, Nakazato H, Ohashi Y. (1999). Adjuvant therapy with oral fluoropyrimidines as main chemotherapeutic agents after curative resection for colorectal cancer: Individual patient data meta-analysis of randomized trials. Jpn J Clin Oncol, 29:78-86.

Tanaka N, Gouchi A, Ohara T, Mannami T, Konaga E, Fuchimoto S, Okamura S, Sato K, Orita K. (1994). Intratumoral injection of a streptococcal preparation, OK-432, before surgery for gastric cancer. Cancer, 74:3097-3103.

Torisu M, Katano M, Kimura Y, Itoh H, Takesue M. (1983). New approach to management of malignant ascites with a streptococcal preparation, OK-432 I. Surgery, 93:357-364.

Watanabe Y, Iwa T. (1984). Clinical value of immunotherapy for lung cancer by the streptococcal preparation OK-432. Cancer, 53:248-253. 